Ying-Jian Lin, Department of Mathematics, Jimei Teacher's College, Xiamen, Fujian 361021, P. R. of China

\title{
ON THE EQUIVALENCE OF McSHANE AND LEBESGUE INTEGRALS
}

\begin{abstract}
This paper gives a new proof of the equivalence of the McShane and Lebesgue integrals, which is independent of measure theory.

The result as stated in the title is well-known. It can be proved in several indirect ways with the aid of the absolute Henstock integral, by using Egoroff's theorem ([3], Chapters 1 and 4) or Lusin's theorem ([1], Sections 2.4 and 2.5), although both of the definitions of the McShane and the descriptive Lebesgue integrals are independent of the measure theory. In this note, following an idea of F. Riesz, we shall give another proof of this result which doesn't rely on measure theory. But the concept of outer measure of an (open) set is necessary. In what follows, let $[a, b]$ be a fixed finite closed interval on the real line, $|\cdot|$ the outer measure of a set and all functions real-valued.

Let us recall the definitions of McShane and Lebesgue integrals and some of their well-known facts which are necessary for our proof. We will refer to $[6],[3],[1],[2]$ and $[4]$ as the references.
\end{abstract}

Definition 1 ([3], Definition 16.7).

1. Let $\delta$ be a positive function defined on $[a, b]$. A finite collection of interval-point pairs $P=\{([u, v], x)\}$ is called a $\delta$-fine McShane partition of $[a, b]$, if the intervals $[u, v]$ in $P$ are nonoverlapping, $\cup[u, v]=[a, b]$ where the union is over $P$, and $[u, v] \subset(x-\delta(x), x+\delta(x))$, where $x$ does not necessarily belong to $[u, v]$.

2. The function $f$ is said to be McShane integrable on $[a, b]$, if there exists a function $F$ with the following property. For each $\epsilon>0$ there exists a

Key Words: McShane integral; Lebesgue integral

Mathematical Reviews subject classification: 26A39

Received by the editors June 22, 1995 
positive function $\delta$ on $[a, b]$, such that $\left|(P) \sum\{f(x)(v-u)-F(u, v)\}\right|<$ $\epsilon$, whenever $P$ is a $\delta$-fine McShane partition of $[a, b]$, where $F(u, v)=$ $F(v)-F(u)$. The function $F$ is called the (McShane) primitive of $f$ and we will write $F(a, b)=(M) \int_{a}^{b} f$.

Definition 2 ([3], Definition 5.1 and the remark after the Theorem 5.7).

1. A function $F$ is said to be absolutely continuous on $[a, b]$, if for every $\epsilon>0$ there exists $\eta>0$ such that for every finite collection of non-overlapping subintervals of $[a, b],\{[u, v]\}$, we have $\sum|F(u, v)|<\epsilon$, whenever $\sum(v-u)<\eta$.

2. A function $f$ is said to be Lebesgue integrable on $[a, b]$ with the (Lebesgue) primitive $F$, if $F$ is absolutely continuous on $[a, b]$ and $F^{\prime}(x)=f(x)$ a.e. in $[a, b]$. We will write $F(a, b)=(L) \int_{a}^{b} f$.

Lemma 1 ([3], Example 16.8; [1], Theorem 2.5.4). If $f$ is continuous on $[a, b]$, then it is McShane integrable on $[a, b]$.

Lemma 2 ([1], Theorem 2.5.6; [3], Theorem 4.1 and the statement in the proof of Theorem 16.10). Let $\left\{f_{n}\right\}$ be a sequence of McShane integrable functions on $[a, b]$. If

1. $f_{n} \rightarrow f(x)$ a.e. in $[a, b]$;

2. $f_{1}(x) \leq f_{2}(x) \leq \ldots \leq f_{n}(x) \leq \ldots$ a.e. in $[a, b]$;

3. $(M) \int_{a}^{b} f_{n}$ converges to $A$ as $n \rightarrow \infty$,

then $f$ is McShane integrable to $A$ on $[a, b]$.

Lemma 3 ([6], Section 46). Let $\left\{f_{n}\right\}$ be a sequence of functions continuous and bounded on the closed subset $E$ of the real line; further suppose that $\left\{f_{n}\right\}$ converges on $E$ as $n \rightarrow \infty$. Then for each $\eta>0$, there is an open set $G$, $|G|<\eta$, such that $\left\{f_{n}\right\}$ converges uniformly on $E \backslash G$.

Lemma 4 ([6], Sections 8, 17 and 23). If $f$ is Lebesgue integrable on $[a, b]$ with the primitive $F$, then so is $|f|$ with the primitive $V_{F}$, where $V_{F}(x)=$ $V(F ;[a, x])$ denotes the total variation of $F$ on $[a, x]$.

Theorem 1 ([5], Theorem 1). If $f$ is McShane integrable on $[a, b]$, then it is Lebesgue integrable on $[a, b]$, and we have $(L) \int_{a}^{b} f=(M) \int_{a}^{b} f$. 
Remark 1 We can give the proofs of the above mentioned lemmas and Theorem 1, without using measure theory.

The following theorem is usually proved indirectly by using Egoroff's or Lusin's theorem. Here we give an alternative proof.

Theorem 2 If $f$ is Lebesgue integrable on $[a, b]$, then it is McShane integrable on $[a, b]$.

Proof. Suppose that $F$ is the Lebesgue primitive of $f$. Then $F$ is absolutely continuous and $F^{\prime}(x)=f(x)$ a.e. on $[a, b]$.

First, suppose $f$ is bounded by $K$. For every positive integer $n$ let $f_{n}(x)=$ $n[F(x+1 / n)-F(x)]$. Then $f_{n}$ is continuous on $[a, b]$ and $f_{n}(x) \rightarrow f(x)$ a.e. in $[a, b]$. Let $\epsilon>0,0<\eta<\epsilon / K$. There is an open set $G^{\prime} \subset[a, b],\left|G^{\prime}\right|<\eta / 2$, such that $\left\{f_{n}\right\}$ converges to $f$ everywhere on $[a, b] \backslash G^{\prime}$. Since each $f_{n}$ is continuous on the closed set $[a, b] \backslash G^{\prime}$ with respect to the set, by Lemma 3 there is an open set $G^{\prime \prime}$ with $\left|G^{\prime \prime}\right|<\eta / 2$, such that $\left\{f_{n}\right\}$ converges uniformly to $f$ on $[a, b] \backslash G$, where $G=G^{\prime} \cup G^{\prime \prime}$ and we have $|G|<\eta$. Note that each $\left\{f_{n}\right\}$ is continuous on $[a, b] \backslash G$ with respect to the set and hence so is $f$. Let $\varphi(x)=f(x)$ when $x \in[a, b] \backslash G$ and linearly elsewhere in $[a, b]$. Then $\varphi$ is continuous on $[a, b]$, and $|\varphi(x)| \leq K, x \in[a, b]$. We have that $\varphi$ is McShane integrable on $[a, b]$ by Lemma 1 . Hence, there is a positive function $\delta$, such that

$$
\left|(P) \sum \varphi(x)(v-u)-(M) \int_{a}^{b} \varphi\right|<\epsilon,
$$

whenever $P$ is a $\delta$-fine partition of $[a, b]$. We may assume that $(x-\delta(x), x+$ $\delta(x)) \subset G$ when $x \in G$. By Theorem $1, \varphi$ is Lebesgue integrable on $[a, b]$ and $(L) \int_{a}^{b} \varphi=(M) \int_{a}^{b} \varphi$. Then we have, for every $\delta$-fine McShane partition $P$ of $[a, b]$,

$$
\begin{gathered}
\left|(P) \sum f(x)(v-u)-(L) \int_{a}^{b} f\right| \\
\leq\left|(P) \sum f(x)(v-u)-(P) \sum \varphi(x)(v-u)\right| \\
+\left|(P) \sum \varphi(x)(v-u)-(M) \int_{a}^{b} \varphi\right|+\left|(L) \int_{a}^{b} \varphi-(L) \int_{a}^{b} f\right| \\
\leq(P) \sum_{x \in G}\{|\varphi(x)|+|f(x)|\}(v-u)+\epsilon+(L) \int_{a}^{b}|\varphi-f| \\
<2 K|G|+\epsilon+2 K|G|<5 \epsilon,
\end{gathered}
$$


where, denoting the open set $G$ by the union of $\left(a_{k}, b_{k}\right), k=1,2, \ldots$,

$$
\begin{gathered}
(L) \int_{a}^{b}|\varphi-f|=\sum_{k}(L) \int_{a_{k}}^{b_{k}}|\varphi-f| \\
\leq \sum_{k}(L) \int_{a_{k}}^{b_{k}}\{|\varphi|+|f|\} \leq \sum_{k} 2 K\left(b_{k}-a_{k}\right)=2 K|G| .
\end{gathered}
$$

Hence $f$ is McShane integrable on $[a, b]$.

Next, let $f$ be unbounded on $[a, b]$. Since $f$ is Lebesgue integrable on $[a, b]$, by Lemma 4 there is no loss of generality assuming $f(x) \geq 0$ for all $x \in[a, b]$. Let $g_{n}(x)=f(x)$ when $f(x) \leq n$ and 0 elsewhere in $[a, b]$. Then $g_{n}$ is bounded and Lebesgue integrable on $[a, b]$. Hence $g_{n}$ is McShane integrable on $[a, b]$ and $(M) \int_{a}^{b} g_{n}=(L) \int_{a}^{b} g_{n}$. Note that $\left\{g_{n}\right\}$ is monotone increasing, and so is $\left\{(M) \int_{a}^{b} g_{n}\right\}$. Furthermore, since $\left\{(L) \int_{a}^{b} g_{n}\right\}$ is bounded by $(L) \int_{a}^{b} f$, we have $\left\{(M) \int_{a}^{b} g_{n}\right\}$ converges. It follows from Lemma 2 that $f$ is McShane integrable on $[a, b]$ and the proof is complete.

Acknowledgment. The author is grateful to the referee for some helpful suggestions for the preparation of this paper.

\section{References}

[1] C. S. Ding and P. Y. Lee, Generalized Riemann integral, Scientific Press, Singapore, 1989, (in chinese).

[2] Y. Kubota, A note on McShanes's integral, Math. Japon., 30 (1985), 5762.

[3] P. Y. Lee, Lanzhou lectures on Henstock integration, World Scientific, Singapore, 1989.

[4] E. J. McShane, A unified theory of integration, Amer. Math. Monthly, 80 (1973), 349-359.

[5] J. Mendoza, On Lebesgue integrability of McShane integrable functions, Real Analysis Exch., 18 no. 2 (1992-93), 556-458.

[6] F. Riesz and B. Sz-Nagy, Leçons d'analyse fonctionelle, 3nd. ed., Akad. Kiadó, Budapest, 1955.

[7] S. Saks, Theory of the integral, 2nd. rev. ed., vol. PWN, Monografie Matematyczne, Warsaw, 1937. 